\title{
The morphogenesis of spatialized cooperation relations: an evolutionary game approach with genetic algorithm
}

\author{
Claude Dupuy ${ }^{1}$ AND ANDRÉ TORRE ${ }^{2}$
}

\begin{abstract}
This paper deals with the morphogenesis of spatialized cooperative relations and with the role of proximity in the evolution, persistence and co-existence of various local strategies. The first part is devoted to a presentation of the analysis of co-operative relations in the evolutionary game theory. In the second part of the paper are presented various simulations on the emergence and evolution of localized co-operative relations, based on endogenous mutations and genetic algorithms. They reveal that a co-operative behavior is a stable, even if complex strategy, and that its emergence and diffusion are favored by the spatial character of inter firms relations. In addition, the results confirm that various strategies can locally coexist and be maintained, generally temporarily.
\end{abstract}

Classification Jel: C5, R1, D2

\section{Introduction}

If the theories on the localization focus on the advantages for a firm to set-up in a production or business area, it is only recently that these theories have integrated the determinants related to the geographical proximity of other producers, in particular the externality factors ( $c f$. Thisse and Torre, 1996), which generate increasing outcomes and non convexities, or the elements of dynamics, which partly contradict the assumption of the existence of footloose firms. The analysis of the specific resources offered by a local area or a local production system hence reveals that it is not sufficient to take into account only the transport or production costs to define the attraction capacities of an area. As regards the temptations to relocate, the regional competition or of the low wages policies, we do consider today the conditions and procedures of a firm setting up, but also its persistence in the long term, in particular in an international context largely independent from any labor law or competition constraints. The persistence of the firm localization depends of course largely on its degree of insertion in the production process,

\footnotetext{
${ }^{1}$ LEREPS Université des Sciences sociales, Manufacture des Tabacs, 21 allées de Brienne, 31042 Toulouse Cedex. E-mail: cdupuy@univ-tlsel.fr

${ }^{2}$ INRA Paris, 147 rue de l'Université, 75007 Paris.
} 
and more particularly on its use of the specific resources and on its ability to build up strong local bonds. We must therefore focus on the dynamic approach, in respect with the projection of the players in a common future by means of convergent anticipations, as well as with the time necessary to set-up co-operative or hierarchic relations.

The subject of this article is the question of the spatialized co-operation relations. This question was the subject of an important literature during the last decade, particularly in the local production systems analysis. Many authors, convinced that one of the keys of the localization persistence resides in the setting up and the preservation of horizontal or vertical connections, underlined the role of the non-market relations in the industrial and territorial construction dynamics. However, only few theoretical elements exist concerning the question of localized co-operative relations, their emergence, their stability or their fading. This article aims at answering this question: to what extend is it possible to set up and to maintain co-operative relations useful for a local long-term setting up? In order to provide an answer, this article is based on simulations taken from the evolutionary games model. It first briefly describes the major evolutionary games models, in particular in the context of the questions of co-operation and space (Sect. 2), before presenting our approach and exposing the results of our model simulations (Sect. 3).

\section{The question of the co-operation at the center of the evolutionary game theory}

The comprehension of the co-operative relations remains particularly important to analyze the methods of coordination between agents, in particular when they are not dependant from rules imposed by a third party such as the State, or from family bonds or religion for example. We know the answers brought by Hobbes to the question of the conflict between the individual and the collective interests which do not obligatorily result from the intentions of the individual actions (the solution consists in imposing a norm by means of a hierarchically higher third party). These solutions explicitly pose the problem of the central feature, even of the existence, of the co-operative relations. In the economic field, the most recent works consisted in a deepening of the nature of the interactions and are based on a reorientation on the face to face bilateral relations between agents. The game theory thus proposes an exploration of the relations between players, by clearly questioning the obtaining of an acceptable solution for "the community" (even if this one is only made of two players). This solution consists, in the static games, in the emergence of one or several Nash Equilibria (NE). But the need for or the interest of these co-operative relations is still an actual question, in respect with the most usually accepted scheme, i.e. the static Prisoner's Dilemma (DP), a game which underlines all the difficulty of a co-operative relation. The co-operation is rejected, even denied.

A more dynamic approach is given by the use of the evolutionary games (Orléan, 1995), in which the strategy determination also rests on the knowledge of the previous behaviors of the players and their repetition and leads to the definition of a new form of equilibrium, the stable fixed points. The repetition possibility of the actions, and thus of multiple and successive meetings, introduced a change in the players strategies. They integrate the need for more friendly "behaviors", would it be only by fear of future 
reprisals. Two model families are generally referred to. Often known in the literature as the dynamic replication model or the stochastic model (Umbauher, 1994), they have the common characteristic to favor the emergence of evolutionary stable strategies (ESS) by the means of a selection process which can be described as short-sighted learning. Indeed, to choose his strategy, each player studies the behavior of the other players in the round $(t-1)$ and adapts according to this observation. In other words, the observation of the payoffs of the other players leads him, with positive probability, to choose the strategy that realized the biggest payoffs in the previous round, with the assumption that its opponents did not modify their strategy in the mean time.

It is possible to introduce mutations in this deterministic frame i.e. perturbations which affect the behavior of the players and modify the simple dynamics of adaptation to the former actions by adding instability factors. These exogenous shocks take the form of a group of mutants, who change brutally their strategy, and this without any experience acquisition of the previous behaviors and any obtained utilities that could predict this change. It can be a change between two already existing strategies, or the emergence of new strategies. In all cases, the mutations create variability, but they take here a deterministic form, the mutation rate: a given proportion of players who move, in each round, from one strategy to an other.

In the initial formulation of the mutations, made by Maynard Smith (1982), only specific and isolated shocks are taken into account, the game evolves then freely until the next shock. In this case, "as the shock has a low amplitude, the system remains around the initial position; and as this shock is isolated, it is the only learning dynamics which then guides the system" (Orléan, 1996). It is easy to show that if the initial position is a stable fixed point, the system will always return to status quo. Foster and Young (1990) precisely examine the resolution of this type of difficulties and underline the stochastic stability criterion (which results from the introduction of stochastic shocks of low amplitude but in continuous time). The stochastic aspect, only present until now in the agent behavior modalities will now be extended to the introduction of random perturbations (Kandori et al., 1993 or Blume, 1994). In all the cases however, (factors of errors, external interventions, or even automatic mechanisms) the mutations considered here are always exogenous, fact that can be considered as a limit for this type of analysis.

\section{The introduction of the endogenous mutations}

The difficulty to build up endogenous mutations concerns the problems of the evolutionary games and appears in the two most often used perturbations analysis methods:

- the evolution dynamic formalization and their variants;

- the attempts at highlighting the evolution processes via simulation procedures.

The formal approach leads to clarify the viability and stability conditions of the systems; it remains however largely unable to provide a coherent description of the endogenous mutations phenomena. A way to circumvent this problem is the use of simulations tests. They makes it possible to carry out the experimentation of various possibilities and to thus have an idea of the results associated with the appearance of various perturbations coming from the players themselves and not from their environment. 
The most known application in terms of simulations is the one carried out by Axelrod (1984). This analysis, which initially excludes all mutation process reveals that the Tit for Tat strategy (TFT) constitutes an ESS, probably because of the capacity of the agents to project themselves in the future and thus to integrate the possibility of successive meetings. It is distrustful by nature since it consists in imitating its opponent strategy. The repetition of the interactions leads here to a prudence which does not appear in a game played only once, so that opportunist temptations are partially neutralized to the benefit of a more benevolent behavior. This result was the subject of an important literature devoted to the analysis of the procedures of co-operation in the case of the iterated DP. This literature shows in particular that the distrustful co-operation, like in TFT, constitutes a better strategy than the unconditional co-operation or than the absolute confidence ( $c f$. Dupuy and Torre, 1997). It can be improved by two means; the first consists in explicitly introducing mutations into the game, the second in using a genetic algorithm approach.

The introduction of the exogenous mutations consists in a relatively simple procedure in the case of an iterated game. It deals with replacing, at each iteration, a part of the existing population by new players presenting different characteristics. The proportion of new players and their strategies are defined by the observer or at random. In the endogenous approach of the mutations, we try to improve this procedure by a change of behavior of the individuals themselves. This operation is based on the application of the genetic algorithms, which explicitly introduce the memory phenomena in the player genotypes. Axelrod (1987) seeks thus to improve its model by using an algorithm of memory 6 (i.e. which integrates, for each player, a memory of the six previous plays), in order to test the robustness of the co-operative solutions in the PD. In a more extended manner, Lindgren attempts to show, in his various works (see for example Lindgren, 1991), how the strategies of the agents endogenously change by using various mutation methods all founded on the memorizing of a succession of rounds played by any agent.

\section{Spatialized games}

Space also plays an important role in the coordination games, because it is partially a product of the interaction between the players, and that it cannot be reduced to the only geonomic distance. It can then be interesting to know to what extend it modifies the processes of coordination between agents.

In the basic model of Maynard Smith, the meetings between agents are the result of a uniform random sample drawn from a given population. Each player can be in contact with any other agent, even if this one is very distant or unknown. However, we know that the economic players generally interact within a given territory and that they can more frequently meet their neighbors. As underlined in the apologs of Schelling (1960), this dimension can play a very important role in the propagation of the opinions, and introduce group or collective effects in the game theory approach. The neighborhood interactions constitute one of the vectors of the new strategy diffusion within a territory (or more largely a network), by the way of imitation, colonization or contamination. They can lead to the formation of local quasi coalitions of players using the same behavior 
rules. The individual is in a frame that can be described as spatial, which results in the existence of privileged relations with the immediate neighbors, the only ones with whom one communicates. The neighborhood is here defined in the broad sense, the individual playing with "close relations", expression of a plural proximity and not only of a geographical one; it generally represents his eight immediate neighbors, or the eight neighbors and himself.

The literature devoted to the spatialized evolutionary games is largely inspired by intuitions noted in the von Neuman theory of the cellular automata. These games were the subject of recent works in the field of economy, in the context of a simple interactions approach (each agent interacts with two neighbors) or of a multiple interaction approach (Berninghaus and Schwalbe, 1996), or of researches more related to the field of the artificial life (Dupuy and Torre, 1997). The principal results of the spatialized games (or lattice games) simulations, in which the players can only maintain relations with their neighbors, shows thus that the introduction of the neighborhood interaction plays an essential and stabilizing role in the strategy development. It seems in particular that proximity (we call proximity the fact to only communicate with his neighbors) favors the emergence of a co-operative evolutionary stable strategy. In other words, a single strategy emerges more easily and its stability is stronger, in spite of the introduction of errors or of exogenous mutations, when agents are close. However, these strategies are fixed in these analyses; what happens to the preceding results if endogenous mutations intervene, mutations which lead to strategy modifications over time? The following paragraphs aim at answering this question.

\section{A spatialized version of the prisoner's dilemma with genetic algorithm ${ }^{1}:$ The emergence and evolution of the cooperative relations}

The evolutionary systems, to which belong the genetic algorithms, appeared at the beginning of the Seventies in Germany and in the United States. Their creation originates in the questioning of the bases of the artificial intelligence, which postulated that the knowledge of the basic rules governing a problem necessarily makes it possible to solve it by inference on these rules. Realizing the application impossibility of such postulates, researchers were then interested in the solutions found in the nature for the resolution of complex problems. The first simulations of spatialized evolutionary games with endogenous mutations were developed by biologists who sought to study the emergence methods of new strategic forms within a population of individuals, the strategies which characterize this type of models were not fixed at the start of the game but had to emerge thanks to a genetic algorithm, and had then to co-evolve. In order to analyze the emer-

\footnotetext{
${ }^{1}$ These simulations have been carried out in co-operation with IRIT (Toulouse) and ENSICA and directed by Hervé Luga and Yves Duthen. For a detailed presentation, see Dupuy C., Duthen Y. and Luga H. (1996) ; Des formes et des jeux, Reading at the conference "Approches Connexionnistes en Sciences Economiques et de Gestion", Nantes.
} 
gence, the evolution and the stability of the co-operative relations in an evolving localized system, two of the operators used in this approach are tested: mutation and selection. The proximity relation is unceasingly privileged, in the sense that the agents preferentially play with their neighbors.

Our simulations are based on a spatialized version of the Prisoner's Dilemma, played in toric universe. They basically differ from the above mentioned works (in particular those of Dupuy and Torre, 1997) since they use a genetic algorithm to make evolve individuals made up in ecosystem. The mutations of this ecosystem are thus endogenous; the system does not undergo any more external shocks but evolves through internal mutations.

The simulation can be split up into two successive phases, which are cyclically repeated:

\section{- A competition phase where the individuals will play K time against their neighbors}

It is the phase of the game itself, which is set-up with intervals of discrete times, so that at the end of $\mathrm{K}$ competitions, a score reflecting the payoffs obtained during the various rounds will be associated to each individual. The adaptation to the environment will then be calculated according to this score, the function of adaptation utilizing the memory of the individuals, linked to the former interactions with each neighbor (Lindgren, 1991) but also to the set of the previous payoffs.

This assumption well corresponds to the purpose of our study: interfirm co-operation in a system of localized production. In such systems, indeed, players have not only one individual memory of the played rounds but they take also account of their collectively evaluated payoffs, for it is not important that individual defections have been expensive in the past if the cumulated payoffs allow a good adaptation on the long term. In this sense, the collective evaluation of the cumulated payoffs reinforces "the benevolence of the players", while guaranteeing a careful attention to the behavior of the close neighbors.

However, the taking into account of the total of the cumulated payoffs implies a strong dependence of the system evolution compared to their distribution over time. For example, if important payoffs were carried out at the simulation beginning thanks to strategies of defection, the co-operators would encounter many difficulties to emerge because of the role played by the former payoffs, which do not encourage a change in strategy. This is why we choose a replication function which records only half of the former payoffs, in order not to give to this variable a too determinant role. In addition, the number of game by generations also plays an interesting role. Simulations presented here are carried out with memory 5 , but the increase in the number of games does not fundamentally modify the results obtained: the emergence of TFT strategies is only delayed in time.

- An evolution phase, during which a genetic algorithm makes the strategies evolve according to their note in the first phase.

Here, the strategy of an individual for a round takes into account the strategies of its eight neighbors at time $t-1$, without needing to individualize them. It consists in a first round, then in a representation of the next round according to the played rounds at 
previous times by the neighbor cells. A round can take the binary value "to defect or to co-operate", the genetic code of each individual thus consists in a binary string of 10 bits, ' 0 ' represents the defection and '1' the co-operation. Whereas the first bit represents the first played round, the nine following bits indicates each round to play according to the number of neighbors who cooperated in the previous round. Thus, if there were 0 co-operator in round $\mathrm{I}$, the round $(i+1)$ corresponds to the second bit of the chain. For 1 co-operator in round $\mathrm{I}$, the round $(i+1)$ will be the third bit of the chain, for 2 co-operators it will be the fourth bit and so on until 8 co-operators. This genetic coding makes it possible to characterize all the possible actions of the individuals, from the most trustful (to cooperate whatever the actions of the neighbors, 11111111) to the more distrustful (00000000), passing through more complex strategies of type 00000001 (co-operation when 7 neighbors cooperate). The ten bits of the genetic code of an individual thus authorize the representation of $2^{10}=1024$ strategies and their differentiation according to their degree of "benevolence". In general, one can analytically draw up five large classes of strategies. The binary chain can evolve thanks to operations of mutations and crossings-over. In order to obtain another generation with potential even better results, the two operations are executed on the current generation. One is to preserve the valueable information stored in the genetic code of the best individuals, the other to create new and different combinations that might prove more successful. The first step of conservation copies a certain percentage of specimens from the parent generation into the new one, without changing their code. The selection of the individuals to be copied is related to their fitness, so that, following the darwinian principle, the better ones have a greater chance of reproduction.

The second step creates new individuals by executing so called genetic operations on selected individuals from the parent generation. These operation may be asexual, like simple mutation or permutation of the genetic code, or sexual, like the most common operation, crossover. In crossover, two individuals are chosen by chance. In the genetic code of each parent, a certain section is randomly chosen. This section may be in different places for both individuals, but must have the same length, since both sections are swapped (crossed other) and reinserted into the respective other parent. The goal of the operation is to combine two partially successful parts into one, more successful whole. The resulting set of individuals now constitutes the next generation and is treated the same way like the first one (execution, measuring fitness and creating a new generation) until a desirable solution has been found or a preset time limit is reached.

The crossing-over operation, which allows an exchange of genetic features between generations, is essential since it basically differentiates the evolutionary systems from the stochastic methods of mutations.

$\begin{array}{lll} & \text { 1010/1101 } & 1010 / 1001 \\ \text { example of crossing-over: } & \text { parent } & \text { Children } \\ & 0110 / 1001 & 0110 / 1101\end{array}$

the mutation randomly acts by modifying a part of the information contained in the data structure of the genotype and makes it possible to create a new individual by the introduction of a new gene. The mutation rate is very low (here $0.5 \%$ ): if it was too high, the system would be completely chaotic, and if it was nil, it would not make possible to 
maintain diversity. Certain strategies, eliminated at the beginning of the game, could be essential thereafter (in fact, this operation artificially maintains the diversity of the system.

$$
\begin{array}{lll}
\text { Mutation example: } & \begin{array}{l}
01101001 \\
(\text { round } t)
\end{array} & \longrightarrow \\
01101101 \\
(\text { round } t+1)
\end{array}
$$

\section{Principal results}

Two of the most significant simulations with regards to the co-operative relations in a local production system are now presented.

a) The first example concerns the analysis of the emergence modalities of the co-operation in an universe initially containing two very simple strategies, to co-operate (co-operation whatever the number of neighbors who cooperate) and to defect (defection whatever number of neighbors which defect), the fixed initial environment being made up of a square small zone $(20 \times 20)$ of co-operators in a $80 \times 80$ universe made of Traitors. We chose to constitute such "a small zone" because an isolated co-operator cannot resist when he is surrounded by traitors.

The results reveal a trend towards proliferation of TFT strategies within the population of the players. Indeed, whereas the initial configuration has $6.4 \%$ of co-operators and $93.6 \%$ of traitors, the co-operation rate ${ }^{2}$ increases to $60 \%$ after 200 generations. One assists, thanks to the genetic algorithm, to the emergence of TFT strategies. During the next generations, the level of co-operation continues to increase, although more slightly, while the average payoffs do not evolve at the same pace. In our simulation, the TFT

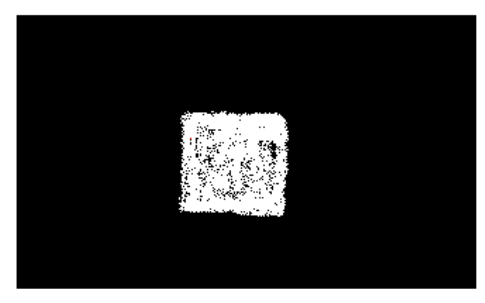

gen $=5$

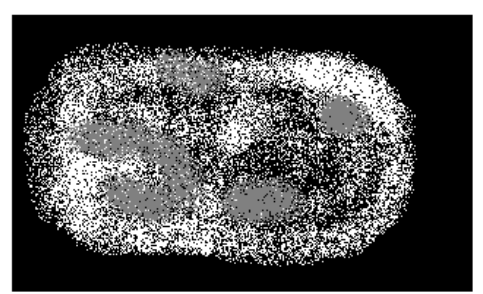

gen $=100$

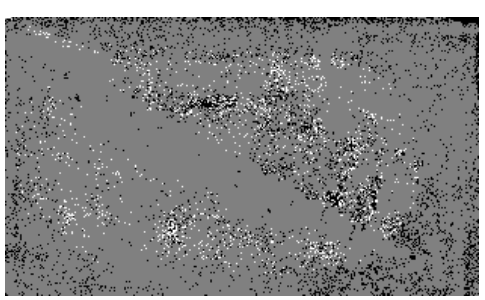

gen $=1000$

Fig. 1. Evolution of the strategies (black: defect; TFT: grey; white: cooperate).

\footnotetext{
${ }^{2}$ Percentage of mutual co-operation (between two neighbors) in the population.
} 
strategies defer from those traditionally used in the evolutionary game theory. Indeed, the rounds are not individualized and each player replies according to the number of co-operators met during the previous round. This procedure is justified by one of our assumptions, the collective character of the strategic choices reinforces "the benevolence of the players", while guaranteeing a close attention to the behavior of the close partners.

The emergence of new strategies is carried out in a certain "order". To the initial cooperators in white (1a), substitute themselves, after 100 generations, confident TFT who initially chose to co-operate (2a). After 200 generations (3a), the ecosystem is far from being stabilized and various forms of TFT coexist with other strategies. One notes thus, in a system characterized by an interaction spatialization and the presence of endogenous mutations, a persistent co-evolution of various strategies. These results, which partially confirm certain simulations carried out with exogenous mutations, show that if the cooperation is a stable strategy, it is not the only one; other strategies can be maintained. The generation 1000 (4a) shows however a total domination of the TFT, which coexist locally with other strategies in a very limited number.

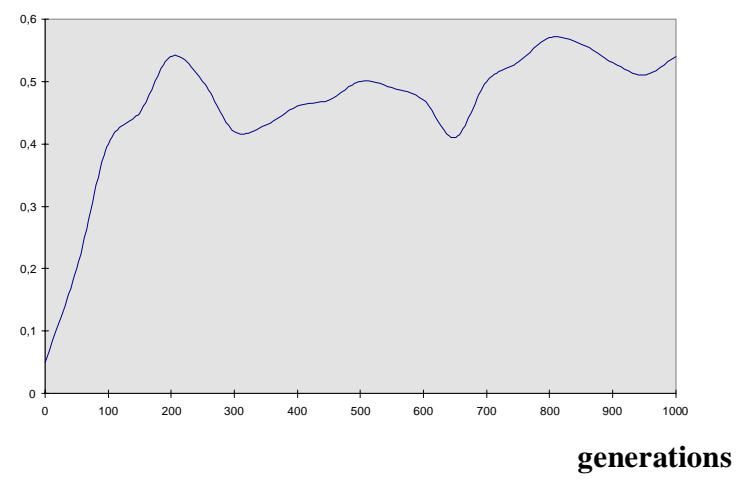

Fig. 2. Evolution of the mutual co-operation (two cells which cooperate).

Thus, in a system characterized by a spatialization, or a non anonymity of the relations in which the history of the interactions plays an important role, strong standardization pressures lead to the emergence of evolutionary stable TFT strategies. In this sense, the proximity contributes to the emergence of the co-operative relations. Should we therefore conclude that all the other strategies will be eliminated? Our simulations show the contrary: out of norm "local strategies" can be maintained and develop, even if their viability is only local and temporary. For example, we can observe small zones of defection, which are maintained over a limited duration. In this sense, proximity plays a double role: by reducing the anonymity of the game, it allows the development of very robust TFT strategies; in addition, in presence of mutation possibilities, other strategies can temporarily appear and be maintained. This is clearly one of the significant results of the iterated Prisoner's Dilemma...

b) The second simulation is founded on an environment initially characterized by the random distribution of various types of strategies. 


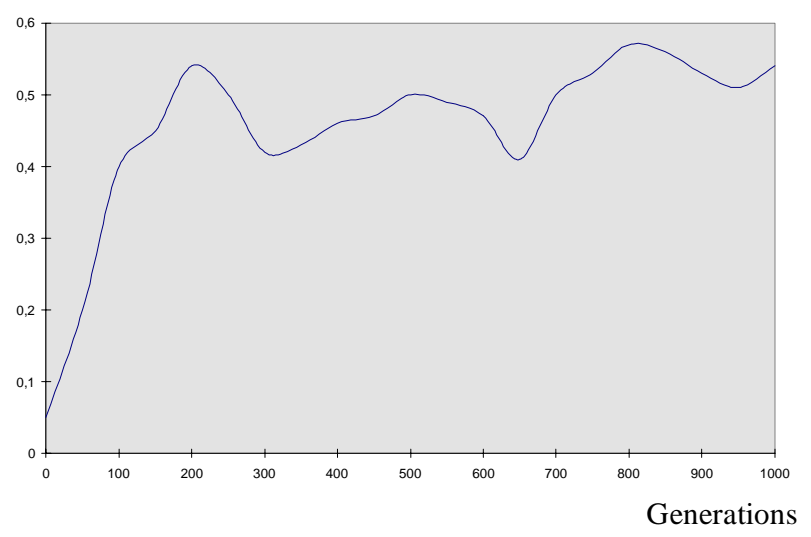

Fig. 3. Evolution of the average payoffs of the population.

One assists, during the first generations, to a brutal fall of the co-operation, due to a chaotic configuration of the system, but the system is thereafter self-organized. During the first periods, only two types of strategies survive: to defect and TFT. Lastly, where mutual co-operation is high, the TFT dominate the others strategies. Once again, the strategy agglomeration or, to be more precise, the spatial character of the interactions, favors the emergence of the co-operative relations.

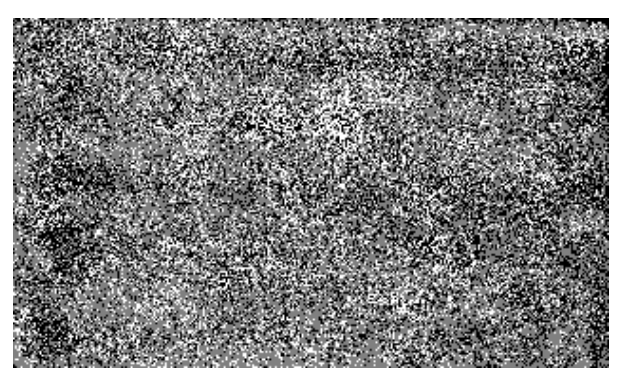

gen $=5$

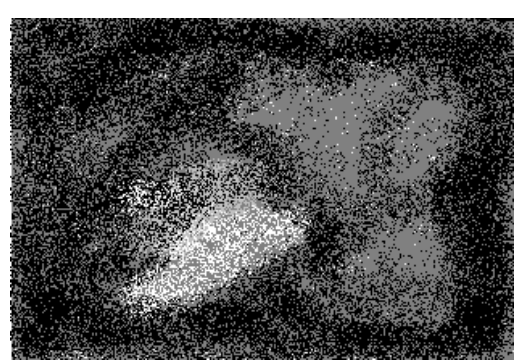

gen $=100$

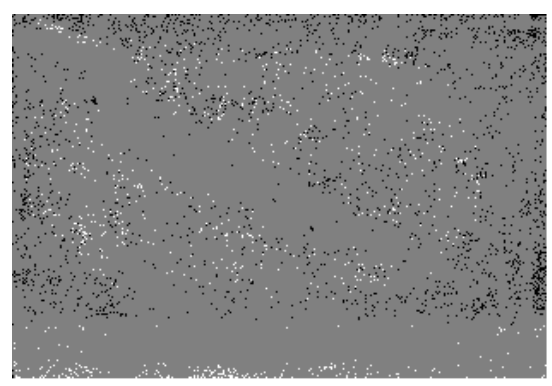

gen $=1000$

Fig. 4. Evolution of the strategies (black: to defect; grey: TFT; white: to cooperate). 


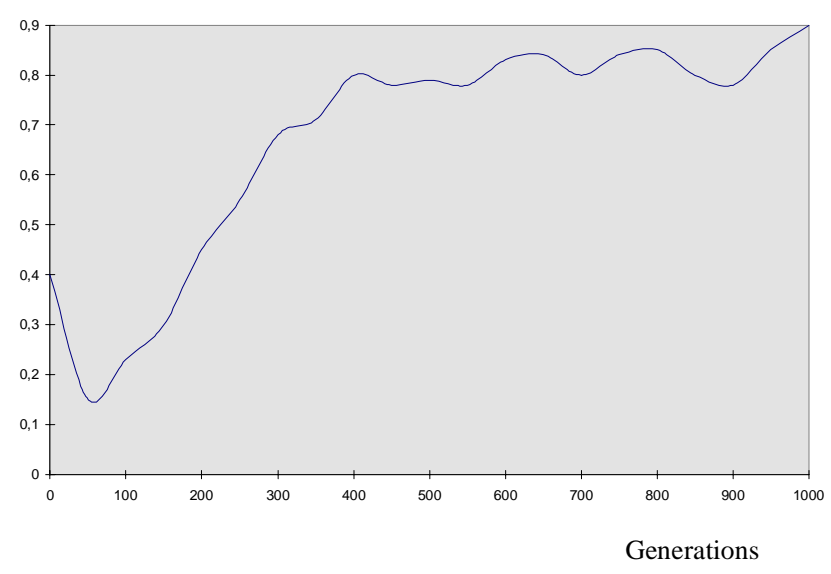

Fig. 5. Evolution of the mutual co-operation (two cells which cooperate).

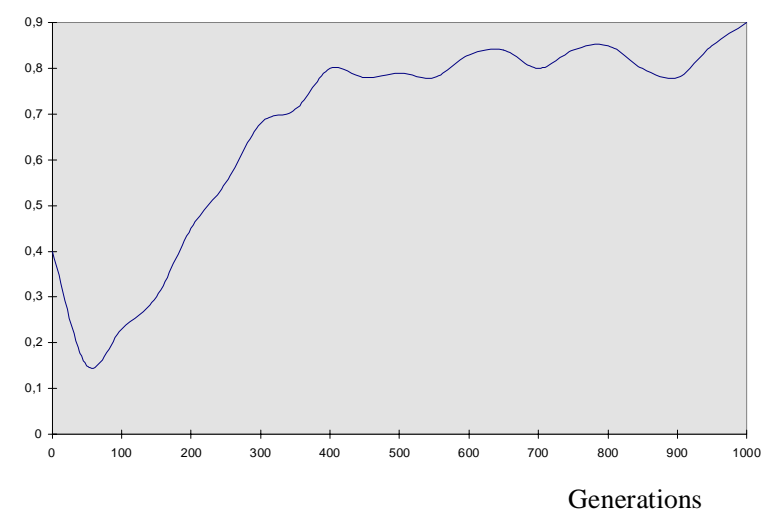

Fig. 6. Evolution of the average payoffs of the population.

\section{Conclusion}

This paper deals with the morphogenesis of spatialized co-operative relations and with the role of proximity in the evolution, persistence and co-existence of various local strategies. The first part is devoted to a presentation of the analysis of co-operative relations in the evolutionary game theory. In the second part of the paper are presented various simulations on the emergence and evolution of localized co-operative relations, based on endogenous mutations and genetic algorithms. They reveal that a co-operative behavior is a stable, even if complex strategy, and that its emergence and diffusion are favored by the spatial character of inter firms relations. In addition, the results confirm that various strategies can locally coexist and be maintained, generally temporarily. 


\section{References}

Axelrod R. (1984) The Evolution of Cooperation. Basic Books, New York.

Axelrod R. (1987) The Evolution of Strategies in the Iterated Prisoner's Dilemma, in Genetic Algorithms and Simulated Annealing, Davis L. (Ed) : Morgan Kaufmann, Los Altos.

Berninghaus S.K., Schwalbe U. (1996) Evolution, interaction and Nash-equilibria, J. Econ. Behav. Organization 29, 57-85.

Blume L.E. (1993) The statistical mechanics of strategic interaction, Games Econ. Behav. 5, 387-424.

Boyer R., Orléan A. (1997) Comment émerge la Coopération ? Quelques enseignements des Jeux Evolutionnistes, in Les limites de la rationalité: Rationalité, éthique et cognition, Jean Pierre Dupuy, Pierre Livet (Eds.), T.1, La Découverte.

Dupuy C., Duthen Y., Luga H. (1996) Des formes et des jeux, Communication au colloque Approches Connexionnistes en Sciences Economiques et de Gestion, Nantes.

Dupuy C., Torre A. (1997) Cooperation and trust in spatially clustered firms, in Learning and Trust in Economy, Lazaric N. et Lorenz E. (Eds.), Edward Elgar Pub., London.

Elster J. (1989) Nuts and Bolts for the Social Sciences, C.U.P., Cambridge Mass.

Foster D., Young H.P. (1990) Stochastics Evolutionnary Game Dynamics, Theoretical Population Biology 38, 219-232.

Holländer J.H. (1990) A Social Exchange Approach to Voluntary Cooperation, American Economic Review 80, 1157-1167.

Kandori M., Mailath G., Roth R. (1993) Learning, Mutation and Long-Run Equilibria in Games, Econometrica 61, 25-56.

Kaneko K. (1989) Pattern Dynamics in Spatiotemporal Chaos: Pattern Selection, Diffusion of Defect, and Pattern Competition Intensity, Physica D 34, 1-41.

Lindgren K. (1991) Evolutionary Phenomena in a simple Dynamics, in Artificial Life II, Langton C.G., Farmer J.D., Rasmussen S. et Taylor C. (Eds): Addison Wesley, Redwood City.

Lindgren K., Nordahl (1993) Evolutionnary dynamics of spatial games, Physica D 40, 40-51.

Maynard Smith J. (1982) Evolution and the Theory of Games, C.U.P., Cambridge Mass.

Nowak M.A., May R. M. (1992) Evolutionnary Games and spatial chaos, Nature 359, 826-829.

Orléan A. (1996) De la stabilité évolutionniste à la stabilité stochastique, Revue Economique 47, 3, 589-600.

Schelling T.C. (1978) Micromotives and Macrobehavior. W.W. Norton and Co, New York.

Thisse J.F., Torre A. (1996) Externalités de Proximité et Localisation Industrielle, Communication au Colloque de l'Association Française de Sciences Economiques, Paris.

Umbhauer G. (1994) Evolutionary Processes and Networks, Mimeo Beta, Univ. de Strasbourg.

Weibull J.M. (1995) Evolutionary Game Theory, MIT Press, Cambridge Mass. 